TRABAJO COMPLETO

\title{
Neumonía grave en la comunidad: ¿cuándo pensar en SAMR-AC?
}

\author{
Recibido: 10/12/18 Aceptado: 17/2/19
}

Elizabeth Di Virgilio, Nicolás Lista, Alejandra Rodríguez, Lautaro de Vedia, Juan Carlos Cisneros, Raúl Prieto.

\section{RESUMEN}

Introducción: Aunque la incidencia de neumonía adquirida en la comunidad (NAC) por Staphylococcus aureus meticilino-resistente adquirido en la comunidad (SAMRAC) es inferior al 10\%, por su elevada mortalidad debe considerarse en los pacientes graves.

Objetivo: Identificar factores de riesgo asociados con SAMR-AC en pacientes con NAC grave.

Material y métodos: Estudio observacional, retrospectivo, que analizó pacientes con NAC con diagnóstico etiológico ingresados en terapia intensiva en un hospital público entre 2006 y 2017.

Resultados: Se incluyeron 250 episodios de NAC, 53 por SAMR-AC y 197 por otros agentes. Los pacientes con SAMR fueron más jóvenes $(35,6 \pm 13,4$ vs $43,1 \pm 12,4$, $\mathrm{p}<0,001)$ y mostraron mayores tasas de infecciones de piel y estructuras relacionadas (IPER) (58,4\% vs $2,0 \%$, $\mathrm{p}<0,001)$, empiema $(15,9 \%$ vs $5,0 \%, p=0,006)$, compromiso radiológico bilateral $(81,1 \%$ vs $36,0 \%, p<0,001)$, promedio de score APACHE II basal $(16,7 \pm 3,8$ vs $13,2 \pm 4,3, p<0,001)$ y requerimiento de ventilación mecánica $(\mathrm{VM})(33,9$ vs 17,6 $p=0,009)$. La tasa de mortalidad fue significativamente mayor para los pacientes con SAMR-AC (35,8\% vs $11,1 \%$, $\mathrm{p}<0,001)$. Las variables que se asociaron con SAMR-AC fueron IPER (OR 67,99, IC 5\% 21,94-210,65), compromiso radiológico bilateral (OR 7,63, IC 95\% 3,67-16,11), score APACHE II $\geq 15$ (OR 4,37, IC 95\% 2,08-9,16), edad $\leq 35$ años
División Asistencia Especial, Departamento de Asistencia Integral al Paciente Infectológico Crítico, Hospital de Infecciosas Francisco J. Muñiz, CABA.

Dirección para correspondencia: elizabethdivirgilio@yahoo.com.ar

Este estudio no tuvo ningún tipo de financiamiento. Ninguno de los autores declara presentar conflicto de intereses en relación a esta publicación.

(OR 3,60, IC 95\% 1,77-7,29), empiema (OR 3,32, IC 95\% 1,24$8,10)$ y VM (OR 2,85 , IC $95 \%$ 1,36-5,86).

Conclusión: En pacientes con NAC grave, la presencia de IPER, compromiso radiológico bilateral, score APACHE $\| \geq 15$, edad $\leq 35$ años, empiema y VM se asociaron significativamente con mayor probabilidad de infección por SAMR-AC.

Palabras clave: Neumonía, neumonía adquirida en la comunidad, Staphylococcus aureus adquirido en la comunidad, SAMR-AC. 


\section{Introducción}

La neumonía adquirida en la comunidad (NAC) es una de las principales causas de muerte por enfermedades infecciosas en todo el mundo (1). En pacientes adultos se estima que la incidencia anual oscila entre 1,07 y 14 casos por cada 1000 personas-año, cifras que no han cambiado en las últimas décadas $(2,3)$. Un estudio realizado en nuestro país y recientemente publicado mostró que, en la ciudad de General Roca, provincia de Río Negro, la incidencia de NAC fue de 7,03 casos (IC 95\%, 6,64-7,44) por 1000 personas-año (4).

La terapia antimicrobiana es esencial en el manejo de la NAC. Los principios en los que se basa la terapia antibiótica inicial de la NAC son la gravedad del cuadro, la probabilidad etiológica y la prevalencia de la resistencia local a los antimicrobianos. Dado que muy pocas neumonías son definidas microbiológicamente en la evaluación inicial del paciente, los médicos normalmente inician el tratamiento antimicrobiano de manera empírica (5).

Entre los agentes bacterianos, Streptococcus pneumoniae representa la causa más frecuente de NAC (6). Sin embargo, en los últimos años se ha registrado un incremento en el número de casos de NAC ocasionada por Staphylococcus aureus resistente a la meticilina adquirido en la comunidad (SAMR-AC) $(7,8,9)$. Un estudio multicéntrico internacional que incluyó datos de 3193 pacientes hospitalizados con NAC de 222 hospitales de 54 países encontró que la prevalencia de SAMR-AC alcanzaba el $3,0 \%$, pero ascendía al $8,1 \%$ cuando se consideraban solo los 1137 casos en que se había obtenido diagnóstico etiológico (10).

A pesar de que su incidencia no parece ser tan elevada, en pacientes graves la presencia de SAMR-AC debe ser adecuadamente considerada, debido a su elevada tasa de mortalidad asociada $(11,12)$. Por lo tanto, hasta que se confirman los resultados de los estudios microbiológicos, debería considerarsela incorporación dela cobertura deSAMR en el régimen empírico, al menos en muchos de los pacientes que cursan con NAC grave $(5,13)$. En consecuencia, resulta de particular importancia reconocer cuáles son las variables específicas que se asocian con un mayor riesgo de padecer una neumonía por SAMR-AC. De esta manera se facilitaría la administración de una terapia antibiótica empírica adecuada, a la vez que se limitaría el uso excesivo de antibióticos con actividad contra SAMR.

\section{Objetivo}

Identificar factores de riesgo asociados a SAMR-AC en pacientes con NAC hospitalizados en unidades de cuidados intensivos (UCl).

\section{Material y métodos}

Se analizaron retrospectivamente todos los episodios de NAC ingresados en la UCI de un hospital público entre 2006 y 2017 en los que el agente etiológico fue identificado. El estudio fue de tipo observacional, sin intervenciones, por lo que los casos se manejaron según las prácticas estándar de cuidados. Los datos de los pacientes permanecen confidenciales y fueron encriptados de manera que no pudiera trazarse hasta el documento fuente, la historia clínica institucional.

\section{Criterios de inclusión}

Pacientes $\geq$ de 18 años de edad que se hospitalizaban en la unidad de cuidados intensivos con diagnóstico de NAC (definido como un nuevo infiltrado pulmonar en la radiografía de tórax realizada al ingreso en el hospital, con signos y síntomas de infección del tracto respiratorio inferior) en los que fue identificado el agente etiológico, ya sea a partir de muestras del árbol respiratorio (esputo, lavado broncoalveolar), como de fuentes extrapulmonares (pleura, hemocultivos). En casos en los que el médico tratante lo considerara se realizaron también serologías para microorganismos atípicos o leptospirosis, hisopado nasofaríngeo para búsqueda de virus respiratorios $\mathrm{y} / \mathrm{o}$ detección de antígenos urinarios para neumococo.

\section{Criterios de exclusión}

Los pacientes no debían haber tenido, durante los últimos doce meses, hospitalizaciones, diálisis, cirugías, admisión en unidades de cuidados crónicos (ej. geriátricos) ni uso de catéteres o elementos médicos que atraviesen la piel.

Se dividió a la población en dos grupos según el agente etiológico: SAMR-AC (grupo SAMR) o no SAMR-AC (grupo No-SAMR). 


\section{Características evaluadas}

Se consideraron las características demográficas (sexo, edad), antecedentes comórbidos (HIV, EPOC, diabetes), presentación clínica (infecciones de piel y estructuras relacionadas [IPER], empiema, compromiso radiológico bilateral, score de APPACHE II, requerimiento de ventilación mecánica) y evolución (mortalidad en el episodio agudo).

\section{Consideraciones estadísticas y plan analítico}

Se utilizó estadística descriptiva: las variables continuas se expresaron en medianas o medias, con desvíos estándar o rango intercuartil, según resultara apropiado, y las variables categóricas se expresaron en porcentajes. Para comparar diferencias entre grupos se utilizaron test de Chi cuadrado o Fisher (variables categóricas) y test de student o suma de rangos de Wilcoxon (variables continuas), según resultara apropiado. Se analizaron diferentes variables demográficas, epidemiológicas y clínicas y se compararon las diferencias entre ambos grupos (análisis univariado). Se realizó análisis de regresión logística de las variables que resultaron significativamente diferentes en el análisis univariado para identificar factores de riesgo asociados con NAC por SAMRAC. Se utilizó estadística descriptiva, test de Fisher para valorar diferencias entre ambos grupos y análisis de regresión logística. Se utilizó el software EPIcalc-2000. Se consideró significativo un valor de $\mathrm{p}<0.05$.

\section{Resultados}

Se incluyeron 250 episodios de NAC, de los cuales $53(21,2 \%)$ fueron causados por SAMR-AC y 197 (78,8\%) por otros agentes. Casi la mitad de los pacientes incluidos (124 de 250, 49,6\%) tenía serología positiva para HIV.

En la Tabla 1 se describen las principales características demográficas, epidemiológicas y clínicas de ambos grupos. Los pacientes del grupo SAMR fueron significativamente más jóvenes ( $35,6 \pm 13,4$ vs $43,1 \pm 12,4$, $p<0,001)$, tuvieron en menorproporción infección por HIV $(24,6 \%$ vs 55,8\%, p<0,001) y presentaron requerimiento de ventilación mecánica (VM) en las primeras 24 horas con mayor frecuencia (33,9 vs 17,6 $p=0,009)$. En cuanto a la presentación clínica, los pacientes del grupo SAMR mostraron tasas significativamente mayores de IPER (58,4\% vs 2,0\%, $\mathrm{p}<0,001)$, empiema $(15,9 \%$ vs $5,0 \%, p=0,006)$ y compromiso radiológico bilateral $(81,1 \%$ vs $36,0 \%, p<0,001)$.
Tabla 1. Características demográficas, epidemiológicas y clínicas de ambos grupos

\begin{tabular}{|c|c|c|c|}
\hline & $\begin{array}{l}\text { NAC por } \\
\text { SAMR }\end{array}$ & $\begin{array}{l}\text { NAC no } \\
\text { SAMR }\end{array}$ & Valor de $\mathrm{p}$ \\
\hline Nro & 53 & 197 & \\
\hline \multicolumn{4}{|c|}{ Características demográficas } \\
\hline $\begin{array}{l}\text { Sexo masculino } \\
\mathrm{N}(\%)\end{array}$ & $40(75,4 \%)$ & $120(60,9 \%)$ & 0,05 \\
\hline $\begin{array}{l}\text { Edad } \\
\text { (Promedio } \pm \mathrm{DE})\end{array}$ & $35,6 \pm 13,4$ & $43,1 \pm 12,4$ & $<0,001$ \\
\hline \multicolumn{4}{|l|}{ Antecedentes } \\
\hline $\begin{array}{l}\text { HIV } \\
\text { N (\%) }\end{array}$ & $14(26,4 \%)$ & $110(55,8 \%)$ & $<0,001$ \\
\hline $\begin{array}{l}\text { Diabetes } \\
\mathrm{N}(\%)\end{array}$ & $2(3,7 \%)$ & $6(3,0 \%)$ & 0,80 \\
\hline $\begin{array}{l}\text { EPOC } \\
\mathrm{N}(\%)\end{array}$ & $2(3,7 \%)$ & $20(10,1 \%)$ & 0,23 \\
\hline \multicolumn{4}{|c|}{ Presentación clínica } \\
\hline $\begin{array}{l}\text { Infección de piel } \\
\text { y estructuras } \\
\text { relacionadas } \\
\mathrm{N}(\%)\end{array}$ & $31(58,4 \%)$ & $4(2,0 \%)$ & $<0,001$ \\
\hline $\begin{array}{l}\text { Empiema } \\
\mathrm{N}(\%)\end{array}$ & $8(15,9 \%)$ & $10(5,0 \%)$ & 0,006 \\
\hline $\begin{array}{l}\text { Infiltrados } \\
\text { pulmonares } \\
\text { bilaterales en } \\
\text { radiografía de } \\
\text { tórax } \\
\mathrm{N}(\%)\end{array}$ & $43(81,1 \%)$ & $71(36,0 \%)$ & $<0,001$ \\
\hline $\begin{array}{l}\text { Score APACHE II } \\
\text { (Promedio } \pm \text { DE) }\end{array}$ & $16,7 \pm 3,8$ & $13,2 \pm 4,3$ & $<0,001$ \\
\hline \multicolumn{4}{|l|}{ Evolución } \\
\hline Óbitos & 19 & 22 & \\
\hline$\%$ mortalidad & $35,8 \%$ & $11,1 \%$ & $<0,001$ \\
\hline
\end{tabular}

SAMR: Staphylococcus aureus meticilino-resistente EPOC: Enfermedad pulmonar obstructiva crónica IPER: Infección de piel y estructuras relacionadas ARM: Asistencia respiratoria mecánica 


\section{Tabla 2. Etiologías de los episodios de NAC}

\begin{tabular}{|l|l|l|}
\hline \multicolumn{1}{|c|}{ Agente etiológico } & \multicolumn{1}{|c|}{$\mathbf{N}(\%)$} & \multicolumn{1}{|c}{$\begin{array}{c}\text { Hemocultivos } \\
\text { positivos N (\%) }\end{array}$} \\
\hline Streptococcus pneumoniae & $110(44 \%)$ & $31(28,1 \%)$ \\
\hline SAMR-AC & $53(21,2 \%)$ & $45(84,9 \%)$ \\
\hline Haemophillus influenzae & $38(15,2 \%)$ & $2(5,3 \%)$ \\
\hline Chlamydia psitacii & $13(5,2 \%)$ & --- \\
\hline Pseudomonas aeruginosa & $8(3,2 \%)$ & --- \\
\hline Klebsiella pneumoniae & $6(2,4 \%)$ & $1(16,6 \%)$ \\
\hline Virus Influenza A H1N1 & $5(2,0 \%)$ & --- \\
\hline Leptospira spp & $3(1,2 \%)$ & --- \\
\hline SAMS & $3(1,2 \%)$ & $2(6,6 \%)$ \\
\hline Moraxella catharralis & $2(0,8 \%)$ & --- \\
\hline Mycoplasma pneumoniae & $2(0,8 \%)$ & --- \\
\hline Otros & $7(2,8 \%)$ & --- \\
\hline
\end{tabular}

SAMR-AC: Staphylococcus aureus meticilino resistente adquirido en la comunidad

SAMS: Staphylococcus aureus meticilino sensible

\section{Tabla 3. Variables asociadas con mayor probabilidad de NAC por SAMR-AC}

\begin{tabular}{|l|l|}
\hline \multicolumn{1}{|c|}{ Variable } & \multicolumn{1}{c|}{ OR (IC 95\%) } \\
\hline $\begin{array}{l}\text { IPER } \\
\begin{array}{l}\text { Compromiso radiológico } \\
\text { bilateral }\end{array}\end{array}$ & $67,99(21,94-210,65)$ \\
\hline $\begin{array}{l}\text { Score de APACHE II } \geq 15 \\
\text { Edad } \leq 35 \text { años }\end{array}$ & $4,63(3,67-16,11)$ \\
\hline Empiema & $3,60(1,77-7,29)$ \\
\hline Requerimiento de ARM & $3,32(1,24-8,10)$ \\
\hline Infección por HIV & $2,85(1,36-5,86)$ \\
\hline
\end{tabular}

SAMR: Staphylococcus aureus meticilino-resistente IPER: Infección de piel y estructuras relacionadas ARM: Asistencia respiratoria mecánica
Los pacientes del grupo SAMR mostraron un promedio de score APACHE $\|$ al ingreso significativamente mayor $(16,7 \pm 3,8$ vs $13,2 \pm 4,3, p<0,001)$, y se asociaron con una tasa de mortalidad significativamente más elevada $(35,8 \%$ vs $11,1 \%, p<0,001)$.

La distribución de las etiologías de NAC se presenta en la Tabla 2. También se incluye en esa tabla la proporción de pacientes en los cuales los hemocultivos fueron positivos.

En la Tabla 3 se exhibe el análisis de regresión logística, el cual mostró que las variables que se asociaron con SAMR fueron la presencia de IPER (OR 67,99, IC 5\% 21,94-210,65), el compromiso radiológico bilateral (OR 7,63, IC 95\% 3,6716,11), un score de APACHE II $\geq 15$ (OR 4,37, IC 95\% 2,08-9,16), una edad $\leq 35$ años (OR 3,60, IC 95\% 1,77-7,29), la presencia de empiema (OR 3,32, IC 95\% 1,24-8,10) y el requerimiento de VM (OR 2,85, IC 95\% 1,36-5,86).

\section{Discusión}

En los últimos años se ha publicado creciente evidencia acerca de que la incidencia de las infecciones invasivas por SAMR-AC está en aumento $(14,15)$. En un trabajo que involucró a 59 hospitales de Estados Unidos entre 2002 y 2003, SAMR fue responsable del $15,9 \%$ de las neumonías y del $8,9 \%$ de las NAC (16). En un estudio multicéntrico llevado a cabo en nuestro país, en el cual se analizaron las infecciones invasivas causadas por SAMR-AC, se vio que en 28 de 55 episodios (51\%) se produjo neumonía (17). Otro estudio, desarrollado en la ciudad de La Plata y publicado en 2014, mostró que SAMR-AC fue causa de 16 casos de NAC sobre un total de 547 , con una incidencia del $2,7 \%$ y una tasa de mortalidad global del 25\% (18).

En un estudio multicéntrico internacional (Global initiative for meticillin-resistant Staphylococcus aureus pneumonia (GLIMP): an international, observational cohort study) (10) en el cual participó nuestro grupo, se evaluaron 3193 pacientes con NAC hospitalizados, encontrándose una prevalencia del $3,0 \%$ para SAMR-AC cuando se consideraba toda la población, pero la misma ascendía al $8,1 \%$ cuando solo se incluía a los 1173 pacientes en los que se pudo determinar la etiología. Sin embargo, en ese estudio, que se realizó en 222 centros de 54 países, se observaron importantes diferencias regionales, e incluso entre los distintos países. En la Argentina, la prevalencia de SAMR-AC fue de 3,4\% (6 casos sobre un total de 179 pacientes) cuando se 
consideraron todos los pacientes, y de $12,2 \%$ entre los casos con diagnóstico etiológico. Si bien esta última cifra no mostró diferencias estadísticamente significativas con el total de los países restantes (7,9\% [89/1124], OR 1,62 (IC 95\% 0,67-3,92, $p=0,28)$, fue la cuarta tasa más elevada entre las 54 naciones participantes (después de Pakistán, Serbia y Estados Unidos).

Los cuadros invasivos ocasionados por SAMR-AC se presentan en general a partir de una IPER, con posterior bacteriemia y una o más localizaciones secundarias (19). En nuestro estudio, el $58,4 \%$ de los casos de NAC por SAMRAC cursó con una IPER, y el 84,9\% tuvieron hemocultivos positivos. Por lo tanto, ante un paciente con NAC, la presencia de una IPER debe orientar hacia la consideración de SAMRAC como posible agente etiológico, tal como se observó en nuestra población.

La tasa de mortalidad asociada a NAC por SAMR-AC parece ser elevada. En una amplia revisión efectuada en los Estados Unidos se reportó una letalidad del $32,4 \%$ (14), mientras que un estudio coreano reportó una tasa de mortalidad del 33\% (20). Otro trabajo, también realizado en los Estados Unidos, encontró que los pacientes con neumonía por SAMR-AC tuvieron un riesgo de morir más de 11 veces mayor (OR 11.34, IC 95\% 5.50-22, p=0.001) en comparación con pacientes con otras infecciones graves por SAMR-AC (21). Por lo tanto, en el tratamiento antimicrobiano empírico de un paciente con NAC no debería faltar la consideración de una posible etiología por SAMR-AC hasta que se cuente con los resultados de los cultivos, particularmente en sujetos jóvenes, con neumonía grave, que cursen con compromiso radiológico bilateral o empiema, o que tengan una IPER concomitante $(13,22)$.

Un estudio prospectivo llevado a cabo en los Estados Unidos, con más de 600 pacientes con NAC, no consiguió identificar ningún factor de riesgo que permitiera predecir qué pacientes podrían tener una neumonía por SAMR-AC. De todos modos, en ese estudio, la tasa de prevalencia de SAMR-AC fue muy baja: $2,4 \%$ en forma global y $5 \%$ cuando los pacientes ingresaban en terapia intensiva (23). Sin embargo, a pesar de esa baja prevalencia, los autores sugieren la inclusión de vancomicina o linezolid en el tratamiento empírico cuando se trata de cuadros con enfermedad muy severa, que ponga en riesgo la vida, tal como recomiendan las guías de IDSA (Infectious Diseases Society of America) para el manejo de las infecciones por SAMR (24).

En otra revisión, que incluyó 943 episodios de NAC, de las cuales 78 fueron por SAMR, se identificaron tres factores de riesgo: antecedentes de infección previa por SAMR en el último año, un elevado score PSI (Pneumonia Severity Index) y administración reciente de antibióticos (20). El estudio GLIMP, antes mencionado, identificó tres factores de riesgo asociados con neumonía por SAMR-AC: infección o colonización previa por SAMR (OR 6,21, IC 95\% 3,25-11,85), infecciones cutáneas recurrentes (OR 2,87, IC 95\% 1,10-7,45) y neumonía grave (OR 2,39, IC 95\% 1,55-3,68).

En nuestro estudio, los resultados muestran que en pacientes con NAC que ingresan en una UCl, la edad $\leq 35$ años, el score de APACHE II $\geq 15$ y la necesidad de VM, junto con la presencia de una IPER, el compromiso radiológico bilateral o empiema, se asociaron significativamente con mayor probabilidad de infección por SAMR-AC. Por el contrario, otros antecedentes como diabetes y EPOC no se asociaron con riesgo de etiología por SAMR. Incluso la infección por HIV pareciera ser un factor protector, aunque este dato debería ser valorado en términos relativos, teniendo en cuenta que la población asistida en nuestra institución, un hospital de referencia en enfermedades infecciosas, tiene una elevada proporción de pacientes con infección por HIV.

Este estudio tiene algunas limitaciones que deben ser mencionadas. En primer lugar, se realizó en un único centro, el cual es a su vez un hospital de referencia especializado en patología infecciosa, por lo que es posible que pueda no representar exactamente lo que se observaría en la población general. De hecho, SAMR-AC fue causa del $21,2 \%$ del total de las NAC con diagnóstico etiológico, una cifra más elevada que la de la mayoría de los centros, posiblemente por tratarse de un centro especializado que recibe derivaciones de otros hospitales. Por otra parte, como ya fue mencionado, el $49,6 \%$ de los pacientes (124/250) tenían serología positiva para HIV, y el $28,1 \%$ (31/110) de las neumonías neumocóccicas fueron bacteriémicas. En segundo lugar, por tratarse de un estudio retrospectivo, está expuesto a sesgos de diversa índole. En tercer lugar, no se incluyó un análisis de apropiabilidad de los tratamientos empíricos, lo cual pudiera haber permitido una mejor comprensión de las diferencias en cuanto a las tasas de mortalidad observadas entre ambos grupos.

La rápida institución de una terapia antibiótica apropiada en pacientes con elevada sospecha de neumonía por SAMR ha sido fuertemente recomendada en varias guías $(24,25,26)$. Las guías de la Sociedad Argentina de Infectología sugieren considerar la cobertura de SAMR en pacientes jóvenes previamente sanos con neumonía grave y rápidamente progresiva, infiltrados cavitados, hemoptisis, influenza previa, usuarios de drogas endovenosas, NAC asociada a rash o leucopenia, infecciones de piel y partes blandas recientes o 
concomitantes, indicando que las opciones de tratamiento incluyen vancomicina, clindamicina o linezolid (5). Debe considerarse que una proporción importante de los pacientes con neumonía por SAMR-AC presenta infiltrados multilobares bilaterales y/o cavitación en los estudios por imágenes, lo cual correlaciona con el examen patológico, que habitualmente revela una neumonía necrotizante hemorrágica con elevados recuentos bacterianos (27). En el presente estudio no pudieron estudiarse todos los pacientes con tomografía computada de tórax a fin de distinguir cuántos de ellos tenían embolias pulmonares sépticas.

La demora en la institución de una terapia antibiótica efectiva fue asociada con un incremento en la mortalidad en pacientes con bacteriemia por SAMR (28). Sin embargo, una terapia de amplio espectro innecesaria puede promover la emergencia de organismos resistentes en la comunidad. Por lo tanto, los datos provenientes de este estudio pueden servir como guía a los médicos prácticos, a fin de intentar morigerar la elevada mortalidad relacionada con la neumonía por SAMR-AC. Por supuesto, el descalonamiento de la terapia antibiótica debería ser considerado inmediatamente de acuerdo a la respuesta clínica del paciente y a los resultados de los cultivos. 


\section{Referencias}

1. The top 10 causes of death, 2016. World Health Organization. Available from https://www.who.int/newsroom/fact-sheets/detail/the-top-10-causes-of-death. Accedido agosto 2018.

2. Torres A, Peetermans WE, Viegi G, Blasi F. Risk factors for communityacquired pneumonia in adults in Europe: a literature review. Thorax 2013;68:1057-65.

3. McLaughlin JM, Johnson MH, Kagan SA, Baer SL. Clinical and economic burden of community-acquired pneumonia in the Veterans Health Administration, 2011: a retrospective cohort study. Infection 2015;43:671-80.

4. Lopardo GD, Fridman D, Raimondo E, Albornoz H, Lopardo $\mathrm{A}$, Bagnulo $\mathrm{H}$, et al. Incidence rate of community-acquired pneumonia in adults: a population-based prospective active surveillance study in three cities in South America. BMJ Open. 2018 Apr 10;8(4):e019439.

5. Lopardo G, Basombrío A, Clara L, Desse J, de Vedia L, Di Líbero E, et al. Neumonía adquirida de la comunidad en adultos. Recomendaciones sobre su atención. Medicina (Buenos Aires) 2015; 75: 245-257.

6. Niederman MS: Community-acquired pneumonia: the U.S. perspective. Semin Respir Crit Care Med 2009, 30:179-188

7. Charlebois ED, Perdreau-Remington $F$, Kreiswirth $B$, Bangsberg DR, Ciccarone D, Diep BA, et al. Origins of communitystrains of methicillin-resistantStaphylococcus aureus. Clin Infect Dis 2004; 39:47-54.

8. Gillet Y,Vanhems P, Lina G, Bes M, Vandenesch F, FloretD,et al. Factors predicting mortality in necrotizing communityacquired pneumonia caused by Staphylococcus aureus containing Panton-Valentine leukocidin. Clin Infect Dis 2007; 45:315-21.

9. Alfaro C, Fergie J, Purcell K. Emergence of communityacquired Staphylococcus aureus in complicated parapneumonic effusions. Pediatr Infect Dis J 2005; 24: 274-276.

10. Aliberti S, Reyes LF, Faverio P, Sotgiu G, Dore S, Rodriguez $A H$, et al. Global initiative for meticillin-resistant Staphylococcus aureus pneumonia (GLIMP): an international, observational cohort study. Lancet Infect Dis. 2016 Dec;16(12):1364-1376.

11. Wunderink RG. How important is methicillin-resistant Staphylococcus aureus as a cause of communityacquired pneumonia and what is best antimicrobial therapy? Infect Dis Clin North Am. 2013 Mar;27(1):17788.

12. Self WH, Wunderink RG, Williams DJ, Zhu $Y$, Anderson EJ, Balk RA, et al. Staphylococcus aureus Community- acquired Pneumonia: Prevalence, Clinical Characteristics, and Outcomes. Clin Infect Dis. 2016 Aug 1;63(3):300-9.

13. Mandell LA, Wunderink RG, Anzueto A, Bartlett JG, Campbell GD, Dean NC, et al. Infectious Diseases Society of America/American Thoracic Society consensus guidelines on the management of community-acquired pneumonia in adults. Clin Infect Dis 2007; 44: Suppl. 2, S27-S72.

14. Klevens RM, Morrison MA, Nadle J, Petit S, Gershman K, Ray S, et al. Invasive methicillin-resistant Staphylococcus aureus infections in the United States. JAMA 2007; 298 : 1763-71.

15. Seybold U, Kourbatova EV, Johnson JG, Halvosa SJ, Wang YF, King MD, et al. Emergence of community-associated methicillin-resistant Staphylococcus aureus USA300 genotype as a major cause of health care-associated blood stream infections. Clin Infect Dis 2006; 42:647-56.

16. Kollef MH, Shorr A, Tabak YP, Gupta V, Liu LZ, Johannes RS. Epidemiology and outcomes of health-care-associated pneumonia: results from a large US database of culturepositive pneumonia. Chest 2005; 128: 3854-62.

17. Fernández $S$, de Vedia L, López Furst MJ, Gardella N, di Gregorio S, Ganaha C et al. Methicillin-resistant Staphylococcus aureus ST30-SCCmec IVc clone as the major cause of community-acquired invasive infections in Argentina. Infect Gen Evol 2013; 14: 401-405.

18. Obed M, García-Vidal C, Pessacq P, Mykietiuk A, Viasus $D$, Cazzola $L$, et al. Clinical features and outcome of community-acquired methicillin-resistantStaphylococcus aureus pneumonia. Enferm Infecc Microbiol Clin. 2014 Jan;32(1):23-7.

19. Castaldo ET, Yang EY. Severe sepsis attributable to community-associated methicillin-resistant Staphylococcus aureus: an emerging fatal problem. Am Surg 2007; 73: 684-7.

20. Jung WJ, Kang YA, Park MS, Park SC, Leem AY, Kim EY, et al. Prediction of methicillin-resistant Staphylococcus aureus in patients with non-nosocomial pneumonia. BMC Infect Dis. 2013 Aug 9;13:370.

21. Wiersma P, D’Angelo MT, Daley WR, Tuttle J, Arnold $\mathrm{KE}$, Ray SM et al. Surveillance for severe communityassociated methicillin-resistant Staphylococcus aureus infection. Epidemiol Infect 2009; 137:1674-8.

22. Woodhead M, Blasi F, Ewig S, Garau J, Huchon G, leven $M$, et al. Guidelines for the management of adult lower respiratory tract infections - Full version. Clin Microbiol Infect. 2011;17:E1-E59. 
23. Moran GJ, Krishnadasan A, Gorwitz RJ, Fosheim GE, Albrecht V, Limbago B, et al. Prevalence of methicillinresistant Staphylococcus aureus as an etiology of community-acquired pneumonia. Clin Infect Dis. 2012 Apr;54(8):1126-33.

24. Liu C, Bayer A, Cosgrove SE, Daum RS, Fridkin SK, Gorwitz RJ, et al. Clinical practice guidelines by the infectious diseases society of america for the treatment of methicillin-resistant Staphylococcus aureus infections in adults and children: executive summary. Clin Infect Dis.2011; Feb 1;52(3):285-92.

25. Nathwani D, Morgan M, Masterton RG, Dryden M, Cookson $B D$, French G, Lewis D:Guidelines for UK practice for the diagnosis and management of methicillin-resistant Staphylococcus aureus (MRSA) infections presenting in the community. J Antimicrob Chemother 2008, 61(5):976994.

26. Rubinstein E, Kollef MH, Nathwani D: Pneumonia caused by methicillin-resistant Staphylococcus aureus. Clin Infect Dis 2008, 46(Suppl 5):S378-385.

27. de Vedia L, Lista N, Piovano G, Amaya Akkuay V, Rodríguez A, Eusebio MJ et al. Staphylococcus aureus meticilino resistente adquirido en la comunidad: una nueva amenaza. Rev Am Med Resp 2012; 4:131-139.

28. Kim S, Park W, Lee K, Kang C, Kim H, Oh M, Kim E, Choe $\mathrm{K}$ : Outcome of Staphylococcus aureus bacteremia in patients with eradicable foci versus noneradicable foci. Clin Infect Dis 2003, 37(6):794-799. 
SEVERE COMMUNITY PNEUMONIA: WHEN TO THINK ABOUT CA-MRSA?

\section{Abstract}

Introduction: Despite the incidence of community-acquired pneumonia (CAP) due to community acquired methicillinresistant Staphylococcus aureus (CA-MRSA) being less than $10 \%$, its presence should be considered in critical patients because of its high rate of mortality.

Objectives: To identify risk factors associated with CA-MRSA in patients with severe CAP.

Materials and method: A retrospective, observational study analysed episodes of etiological diagnosis in patients admitted in Intensive Care Unit in a public hospital between 2006 and 2017.

Results: 250 episodes of NAC were included, among which 53 were caused by SAMR-AC and 197 by other agents. Patients with MRSA were the youngest $(35.6 \pm 13.4$ vs $43.1 \pm 12.4$, $\mathrm{p}<0.001)$, and showed higher rates of skin and skin-structure infections (SSSI) (58.4\% vs 2.0\%, p<0.001), empyema ( $15.9 \%$ vs $5.0 \%, \mathrm{p}=0.006)$, bilateral radiological compromise $(81.1 \%$ vs $36.0 \%, p<0.001)$, average base-line APACHE II score $(16.7 \pm 3.8$ vs $13.2 \pm 4.3, p<0.001)$ and mechanical ventilation requirement rate (MV) (33.9 vs $17.6 \mathrm{p}=0.009)$. The mortality rate was significantly higher than the one in CA-MRSA patients ( $35.8 \%$ vs $11.1 \%, \mathrm{p}<0.001)$. The variables associated with CA-MRSA were SSSI (OR 67.99, IC 5\% 21.94-210.65), bilateral radiological compromise (OR 7.63, IC 95\% 3.6716.11), APACHE II score $\geq 15$ (OR 4.37, IC 95\% 2.08-9.16), age $\leq 35$ years (OR 3.60, IC 95\% 1.77-7,29), empyema (OR 3.32, IC 95\% 1.24-8.10) and MV (OR 2.85, IC 95\% 1.36-5.86).

Conclusion: The presence of SSSI, bilateral radiological compromise, APACHE II score $\geq 15$, age $\leq 35$ years, empyema and $\mathrm{MV}$ in patients with severe CAP was largely associated with higher probability of CA-MRSA infection.

Key words: Pneumonia. Community acquired pneumonia. Community acquired Staphylococcus aureus. CA-MRSA. 